\title{
Gleason inflation 1998-2011: a registry study of 97168 men
}

\section{Daniela Danneman, Linda Drevin*, David Robinson ${ }^{\dagger}$, Pär Stattin and Lars Egevad}

Department of Oncology-Pathology, Karolinska Institutet, Stockholm, *Regional Cancer Centre, Uppsala University Hospital, Uppsala, ${ }^{\dagger}$ Department of Urology, Ryhov County Hospital, Jönköping, ${ }^{\ddagger}$ Department of Surgery and Perioperative Sciences, Urology and Andrology, Umeå University, Umeå, Sweden

\section{Objectives}

To study long-term trends in Gleason grading in a nationwide population and to assess the impact of the International Society of Urological Pathology (ISUP) revision in 2005 of the Gleason system on grading practices, as in recent years there has been a shift upwards in Gleason grading of prostate cancer.

\section{Patients and Methods}

All newly diagnosed prostate cancers in Sweden are reported to the National Prostate Cancer Register (NPCR). In 97168 men with a primary diagnosis of prostate cancer on needle biopsy from 1998 to 2011, Gleason score, clinical T stage (cT) and serum levels of prostate-specific antigen (s-PSA) at diagnosis were analysed.

\section{Results}

Gleason score, cT stage and s-PSA were reported to the NPCR in $97 \%, 99 \%$ and $99 \%$ of cases. Before and after 2005 , Gleason score $7-10$ was diagnosed in $52 \%$ and $57 \%$, respectively $(P<0.001)$. After standardisation for $\mathrm{cT}$ stage and s-PSA with 1998 as baseline these tumours increased from $59 \%$ to $72 \%$. Among low-risk tumours (stage cT1and s-PSA 4-10 ng/mL) Gleason score $7-10$ increased from $16 \%$ in 1998 to $40 \%$ in
2011 ( $P$ trend $<0.001$ ), mean $19 \%$ and 33\% before and after $2005(P<0.001)$. Among high-risk tumours (stage T3 and s-PSA $20-50 \mathrm{ng} / \mathrm{mL}$ ) Gleason score 7-10 increased from $65 \%$ in 1998 to $94 \%$ in 2011 ( $P$ trend $<0.001$ ), mean $78 \%$ and $90 \%$ before and after $2005(P<0.001)$. A Gleason score of $2-5$ was reported in $27 \%$ in 1998 and $1 \%$ in 2011. Gleason score 5 decreased sharply after 2005 and Gleason score 2-4 was almost abandoned.

\section{Conclusions}

There has been a gradual shift towards higher Gleason grading, which started before 2005 but became more evident after the ISUP 2005 revision. Among low-stage tumours reporting of Gleason score 7-10 was more than doubled during the study period. When corrected for stage migration upgrading is considerable over recent decades. This has clinical consequences for therapy decisions such as eligibility for active surveillance. Grading systems need to be as stable as possible to enable comparisons over time and to facilitate the interpretation of the prognostic impact of grade.

\section{Keywords}

needle biopsy, pathology, prostate cancer, Gleason grade

\section{Introduction}

Almost 50 years have passed since the introduction of the Gleason grading system and it is still one of the most powerful prognostic and predictive factors in prostate cancer [1-4]. In 2005, Gleason grading underwent its first major revision at an International Society of Urological Pathology (ISUP) consensus conference [5]. A discrepancy between Gleason's original criteria and how grading was applied in practice had been noted [6]. Definitions of Gleason patterns and some rules for reporting of grade data were unclear and there was a need of standardisation. Among the most important changes were inclusion of cancers with poorly formed glands and most cribriform cancers in the Gleason pattern 4. It was later suggested that all cribriform glands should be diagnosed as
Gleason pattern 4 [7]. Further, in needle biopsies, the definition of Gleason score was modified to include any component of higher grade $[5,8]$. Prostate biopsy protocols have become more extensive over the last decade but still only sample a small proportion of the tumour. Even with extended protocols there is a risk that the biopsy grade is not representative of the entire tumour. One of the purposes of the ISUP revision was to ensure that a high-grade component is included in the diagnosis even if minute, as there may be a more substantial amount of high-grade cancer in the radical prostatectomy (RP) specimen.

There have been several reports of a shift upwards of Gleason grading over recent decades [9-13]. Most of these reports have investigated trends before ISUP 2005 [9-12]. 
However, it can be supposed that ISUP consensus recommendations have contributed to this development [13-18]. There has been a concurrent shift towards lower stages and lower serum PSA levels at diagnosis, which may mask some of the upgrading and the true Gleason inflation can thus be even greater that previously reported. Changed grading practices affect treatment decisions, notably the eligibility for active surveillance (AS). Most AS protocols exclude patients with Gleason scores of $\geq 7$ [19-23]. There is an increasing awareness that patients with low-risk disease have a low risk of dying from prostate cancer during the first 10 years after diagnosis [24] and even longer [25]. Therefore, an accurate assessment of the Gleason score is critical for avoiding overtreatment. The objective of the present study was to investigate long-term trends in Gleason grading of prostate biopsies in a nationwide population. We particularly aimed to assess the impact of ISUP 2005 on grade reporting.

\section{Patients and Methods}

Histopathological and clinical data and primary treatment of all newly diagnosed prostate cancers in Sweden are reported to the National Prostate Cancer Register (NPCR) since 1998 [26]. Between 1998 and 2011, 97168 men were diagnosed with prostate cancer based on assessment of core biopsies from TRUS-guided needle biopsies. Gleason score, Gleason patterns, clinical T stage (cT) and serum levels of PSA (s-PSA) at diagnosis were analysed.

Direct standardisation of the Gleason score was used to adjust for the changing distributions of $\mathrm{cT}$ stage and s-PSA during the study. The distribution in 1998 was used as the standard with the following categorisations: four levels of cT stage (T1, T2, T3, and T4) were combined with three levels of s-PSA (above or below median s-PSA or missing value).

To evaluate whether there was a change in Gleason score trend in 2005, the association between the proportion Gleason score 2-6 and year of diagnosis was modelled using logistic regression. Adjustment was made for $\mathrm{cT}$ stage and s-PSA, using the same categorisation as above. Two such models, one with and one without broken-line regression, were compared using the chi-square test in order to see if the broken-line model better explained the data.

Trend tests were performed using logistic regression, with year of diagnosis as the continuous variable and the proportion of interest as the outcome. Such a trend test was also used to evaluate the proportion of Gleason score $3+4=7$ among Gleason score 7 tumours. The chi-square test was used to compare the two time periods after standardisation. The Mann-Whitney test was used to compare the median s-PSA in 1998 and 2011. All tests were two-sided and a $P<0.05$ was considered to indicate statistical significance. The statistical analyses were performed using the R statistical program package (version 2.15.1).

\section{Results}

Gleason score, cT stage and s-PSA were reported to NPCR in $97 \%, 99 \%$ and $99 \%$ of the 97168 cases. In the early years of the NPCR, the total Gleason score was reported but not always the Gleason patterns. From the year 2000 the primary and secondary Gleason patterns were reported in $75 \%$ of Gleason score 7 and from 2007 they were always reported.

The distribution of Gleason score from 1998 to 2011 is shown in Table 1. Before and after 2005, Gleason score 7-10 was diagnosed in $52 \%$ and $57 \%$, respectively $(P<0.001)$. The proportion of cancers diagnosed with a Gleason score 6 increased until 2004-2005, when there was a trend shift and a decline began (Fig. 1A). The percentage of Gleason score 7 increased during the entire study period. Tumours assigned a Gleason score 8 or 9 also increased; while the proportion of rare Gleason score 10 cancers remained more stable.

The use of Gleason score 5 decreased sharply after 2005 and Gleason score $2-4$ is now almost entirely abandoned (Fig. 1A). A Gleason score of 2-5 was reported in $27 \%$ in 1998 and in $1 \%$ in 2011 (odds ratio [OR] $0.80, P_{\text {trend }}<0.001$ ). A Gleason score of $8-10$ was reported in 20\% in 1998 and $22 \%$ in 2011 (OR 1.01, $\left.P_{\text {trend }}=0.006\right)$. When adjusted for stage and s-PSA, Gleason score $8-10$ increased from $20 \%$ in 1998 to $36 \%$ in 2011 (OR 1.06, $P_{\text {trend }}<0.001$ ).

The proportion of Gleason score $3+4=7$ vs $4+3=7$ among Gleason score 7 tumours in 2000-2011 is shown in Figure 2. For most reported years $>60 \%$ of Gleason score 7 was Gleason score $3+4=7$ (range 57-64\%). There was a weak trend towards increasing proportion of Gleason score $3+4=7$ over the period (OR 1.01, $\left.P_{\text {trend }}=0.02\right)$.

Newly diagnosed cancers were stage T1c in 20\% in 1998 and $51 \%$ in 2011, while T2 and T3 stages decreased from $40 \%$ and $31 \%$, respectively in 1998 to $29 \%$ and $16 \%$, respectively in 2011 $\left(P_{\text {trend }}<0.001\right)$. There was a concurrent decrease in the median s-PSA at diagnosis with a reduction from $20.5 \mathrm{ng} / \mathrm{mL}$ in 1998 to $8.9 \mathrm{ng} / \mathrm{mL}$ in $2011(P<0.001)$.

A broken line regression analysis of change of distribution of Gleason score over time showed a slightly steeper decrease in the use of Gleason score 2-6 after $2005(P=0.007)$.

Before and after 2005, Gleason score 7-10 was diagnosed in $52 \%$ and $57 \%$, respectively $(P<0.001 ;$ Fig. $1 \mathrm{~A})$. With a stage migration towards lower stages at diagnosis during the study period, we would expect lower Gleason scores at diagnosis. Therefore, Gleason score distributions were standardised for cT stage and s-PSA with 1998 as baseline (Fig. 1B). After 




standardisation, Gleason score 7-10 tumours were diagnosed before and after 2005 in 59\% and 72\%, respectively $(P<0.001$; Fig. 1B).

Among low-risk tumours (stage T1c and s-PSA 4-10 ng/mL), Gleason score $7-10$ increased from $16 \%$ in 1998 to $40 \%$ in 2011 (OR 1.12, $P_{\text {trend }}<0.001$ ), with a mean of $19 \%$ and $33 \%$ before and after 2005, respectively $(P<0.001$; Fig. 3a).

Among high-risk tumours (stage T3 and s-PSA $20-50 \mathrm{ng} / \mathrm{mL}$ ) Gleason score $7-10$ increased from $65 \%$ in 1998 to $94 \%$ in 2011 (OR 1.16, $P_{\text {trend }}<0.001$ ), with a mean of $78 \%$ and $90 \%$ before and after 2005, respectively ( $P<0.001$; Fig. 3B). The increase in the group of Gleason score 8-10 was sharpest from 2004.

There were small variations in grading practices among the six healthcare regions of Sweden. In all regions the percentage of Gleason score 7-10 increased from 1998-2004 to 2006-2011 and Gleason scores of 2-5 were almost entirely abandoned (Fig. 4).

\section{Discussion}

The 2005 ISUP revision of the Gleason grading system introduced new sets of rules for interpretation of morphological patterns and for reporting of grade data. All of these changes have the potential to cause a shift towards higher grading. Several investigators have observed a trend towards increasing prostate cancer grades over recent decades $[10,10,11,11,12,12,14,16]$. It is unclear how much the ISUP revision has contributed to this process. The present study period from 1998 to 2011 spans several years before and after the release of the ISUP recommendations. The data thus enable an analysis both of long-term trends and the impact of the ISUP revision.

Several investigators have compared conventional and modified Gleason grading by re-grading biopsies [14-18,27]. In these studies, the proportion of needle-biopsy cancers with Gleason score $\geq 7$ increased from $36.7 \%$ to $77.7 \%(n=368)$ [15], from $31.4 \%$ to $50.6 \%(n=172)$ [14], from $53.4 \%$ to $59.2 \%(n=103)$ [18], from $89.1 \%$ to $95.4 \%(n=590)$ [27], from $38.1 \%$ to $49.5 \%(n=97)$ [17] and from $64.6 \%$ to $68.5 \%$ $(n=565)$ [16]. Kuroiwa et al [16] used the new pattern interpretation rules in both datasets and thus only analysed the impact of the ISUP grade reporting rules. Several of these studies are limited by small cohort sizes. A few studies have compared grading practices before and after the ISUP revision in larger clinical series $[13,28]$. Zareba et al [13] reported an increase of cancers with Gleason score $\geq 7$ from $32.0 \%$ in 2000-2004 to $45.2 \%$ in 2005-2007.

In the present study, for the first time, we investigated the impact of the ISUP 2005 revision of Gleason grading in a national cohort. Consistent with previous studies we found a significant inflation of Gleason grading during the study 
Fig. 1 (A) Gleason scores from 1998 to 2011. (B) Gleason scores standardised for CT stage and s-PSA.
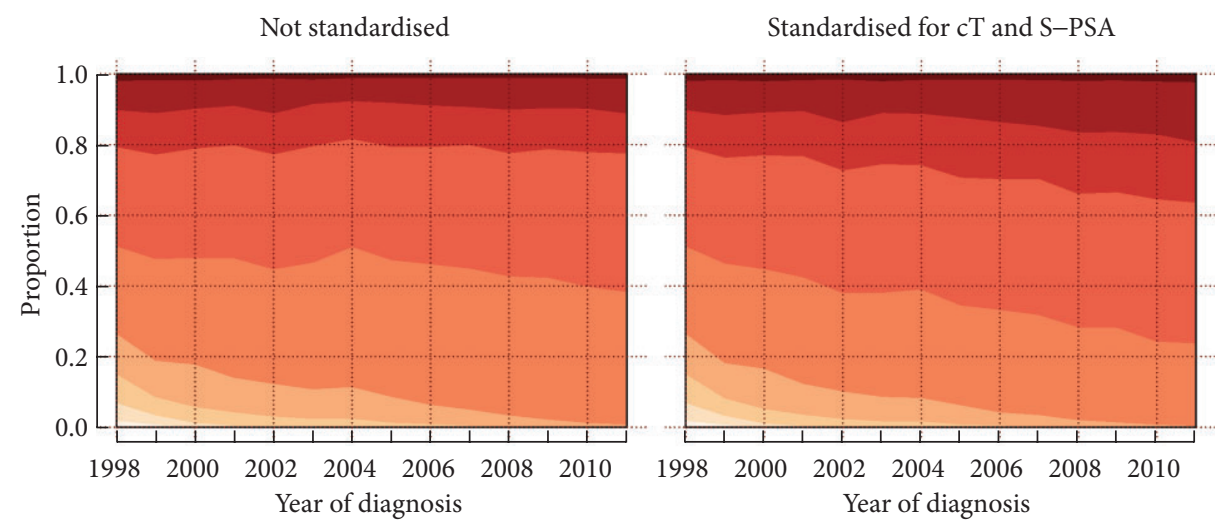

Standardised for cT and S-PSA

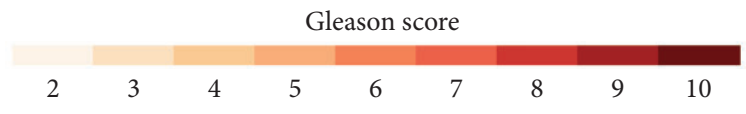

Fig. 2 Distribution of Gleason score 3+4 = 7 and 4+3 = 7 among Gleason score 7 tumours between 2000 and 2011 .

Distribution of Gleason score $3+4=7$ and $4+3=7$ among Gleason score 7 tumours

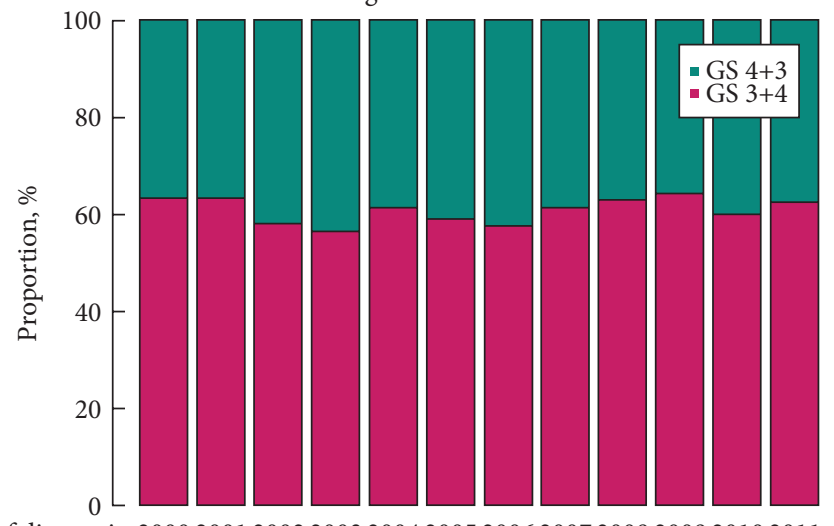

Year of diagnosis: 200020012002200320042005200620072008200920102011

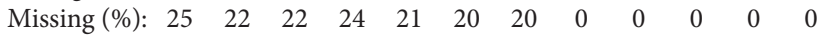

period. This has been a gradual process that started several years before the ISUP consensus conference but became more evident after 2005.

Gleason patterns 1 and 2 in needle biopsies and RP specimens are now almost never diagnosed. Thus, Gleason scores $2-5$ are hardly used at all, similar to reports of others [10]. This most probably has little clinical relevance, as the diagnosis of Gleason score $2-5$ vs 6 should not affect the treatment decision.

A more important observation is the decrease of Gleason score 6 tumours and concurrent increase of Gleason score 7-10 cancers. The distribution among these grades is important for deciding which patients should be offered primary treatment with curative intention vs AS. In most AS programmes a Gleason score $\geq 7$ excludes the patient from AS [20]. If tumours are overgraded because some pathologists have become eager to diagnose even minimal findings of poorly formed or fused glands as Gleason score 7, there is a risk of overtreatment of indolent tumours. There is a justified concern among urologists that the clinical relevance of Gleason score 7 will be diluted when too many low-risk tumours are moved into this category. It was recently proposed that Gleason score 6 tumours would not be cancers based on the observation that cancers with lymph node metastases are invariably of higher grade [29]. This is not a generally accepted concept, but it underscores the obvious need to better define the Gleason score 6 category. The distinction between Gleason score $3+4=7$ and $4+3=7$ is also clinically important as these have different prognosis. The grade distribution within Gleason score 7 remains remarkably stable with slightly more than $60 \%$ of Gleason score 7 cases still being diagnosed as Gleason score 3+4 $=7$ (Fig. 3).

An increasing incidence of low-risk tumours and a concomitant decrease of high-risk tumours at diagnosis was seen, similar to that reported by others [30]. This reflects a stage migration with cancer being detected at earlier stages. As stage and grade correlate this should actually cause a shift towards lower grades at diagnosis. Yet, we see an upgrading both among all cancers and among low-risk cancers. Some of the Gleason inflation is masked by this concurrent trend towards lower stages and lower s-PSA at diagnosis. When corrected for stage and s-PSA the upgrading becomes more evident.

We think that Gleason upgrading has been both a gradual process of changing practices in the pathology community and a consequence of the ISUP 2005 revision. Some changes in grading routines result from improved understanding of tumour biology. Some Gleason pattern 1 cases may have been adenosis, i.e. benign proliferations, and there is no evidence that tightly packed small cancer glands would have different clinical behaviour than other Gleason pattern 3 cancers. In the early works by Gleason, small rounded cribriform glands 


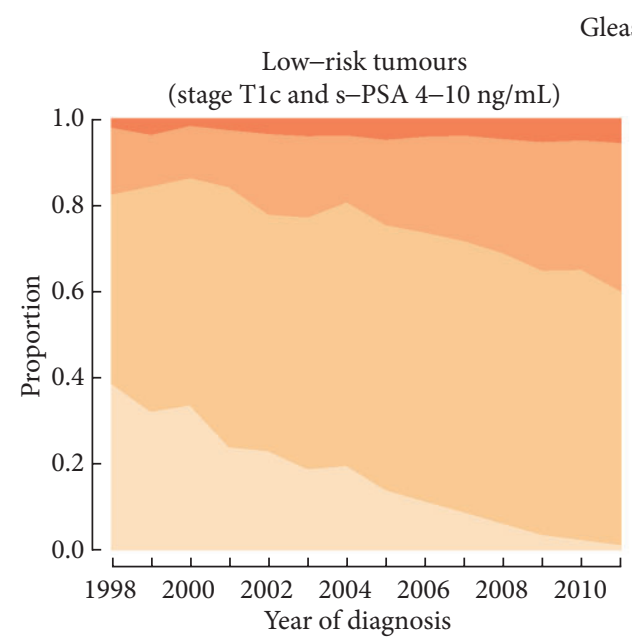

Gleason score

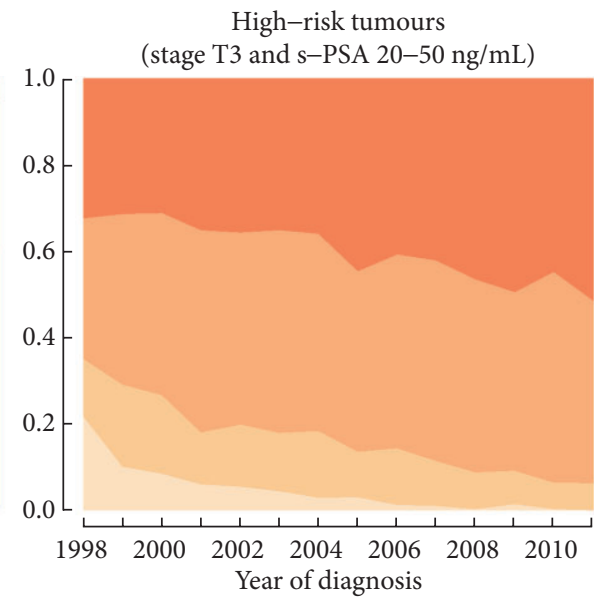

Gleason score

\begin{tabular}{rlrr}
\multicolumn{5}{l}{ Gleason score } \\
\hline $2-5$ & 6 & 7 & $8-10$
\end{tabular}

Fig. 4 Proportions of Gleason scores between 1998 and 2011 in six Swedish healthcare regions.
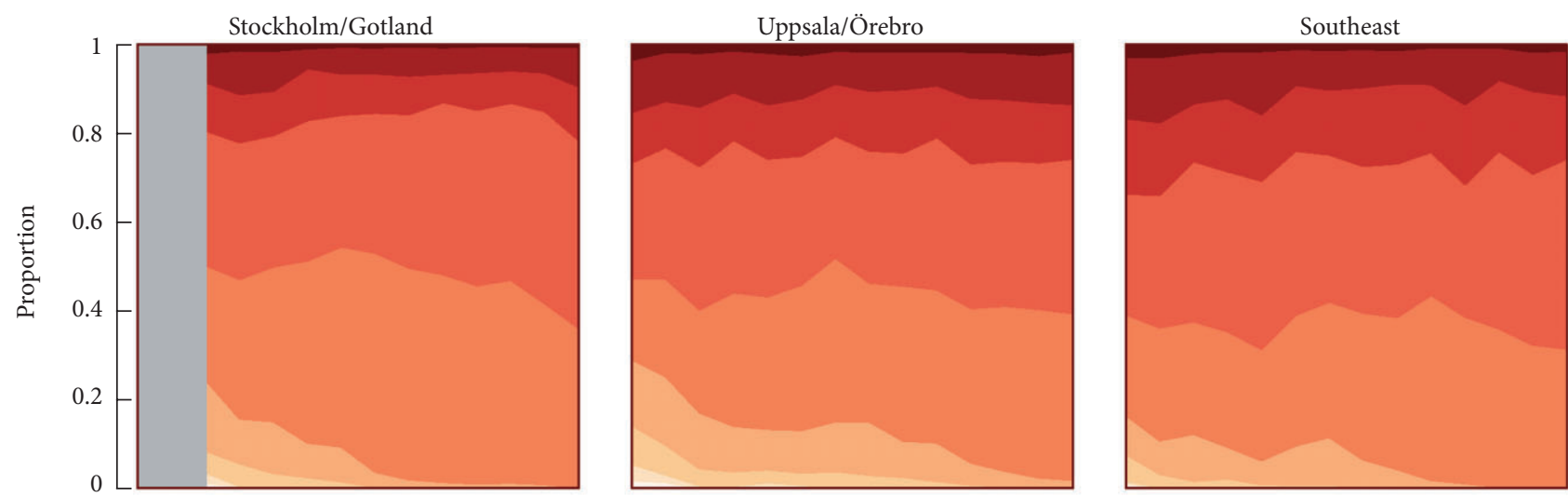

Gleason

score
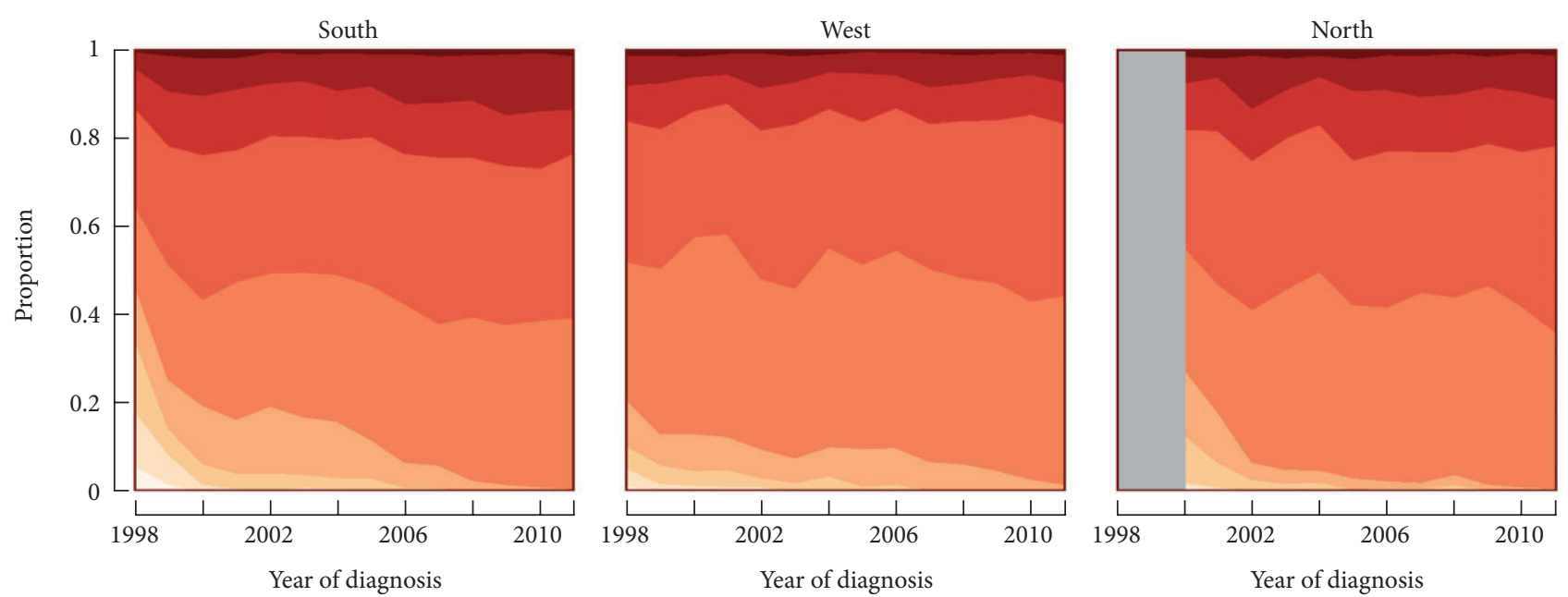

(stage Tlc and s-PSA 4-10 ng/mL), 1998-2011.

(B) Gleason score, high-risk tumours (stage T3 and s-PSA $20-50 \mathrm{ng} / \mathrm{mL}$ ), 1998-2011. 
were part of the Gleason pattern 3 and in some of his microphotographs cribriform patterns were even seen in Gleason pattern 2 [3]. There is now evidence that cribriform cancer is an aggressive disease, which justifies the decision to include it in Gleason pattern $4[31,32]$. Other changes are more arbitrary. In studies comparing grading of experts and general pathologists the expert grading has almost invariably been more aggressive. In the end, aggressive grading could be perceived as a sign of expertise and this fear of undergrading may have driven the process.

Alternative explanations of changing grade distribution over time include changed tumour biology due to lifestyle changes, but this is unlikely to occur within less than two decades. Prostate biopsies underestimate tumour grade even with the ISUP revision of Gleason grading. Biopsy protocols have changed to become more extensive, which increases the likelihood of finding a high-grade component. However, in one study grading accuracy only improved from $43.5 \%$ to $45.2 \%$ by going from a sextant protocol to an average of 10 biopsies [33]. There is evidence that some grade progression occurs in the natural history of prostate cancer as seen in repeat biopsies in patients on AS. It has been modelled that grade progression during AS would occur in $12-24 \%$ during a 10-year period [34]. However, in the present study data from the primary diagnosis was used and if the grade progressed in patients on AS, it would not affect the study results. Thus, the main reason for the significant Gleason inflation is most probably a changed grading practice among pathologists.

There were small variations in grading practice among the six healthcare regions of Sweden. In all regions there was a similar trend, with a shift upwards of Gleason grading, indicating that the modifications of Gleason grading have been well disseminated and adopted throughout the country. Several efforts have been made to standardise prostate pathology in Sweden [35]. Images were circulated among all Swedish pathologists immediately after the ISUP consensus conference, with the intention of informing and explaining the ISUP modification of the Gleason grading system. Furthermore, most Swedish pathologists have been instructed in prostate pathology at multi-headed microscope tutorials during the study period, which has also contributed to a high level of grading uniformity. This emphasises the importance of regular and systematic training for standardised grade reporting.

Before the ISUP revision, exact concordance between biopsy and RP specimen Gleason score was found in $28-68 \%$ (pooled data: $44.5 \%$ ) [33]. The biopsies undergraded in $24-60 \%$ (pooled 45\%) and overgraded in 5-32\% (pooled 10.4\%). An argument for introducing modified Gleason grading was that it would improve this concordance by reporting even minimal areas of high-grade cancer on biopsy. Several groups have addressed the correlation between Gleason score in needle biopsies and RP specimens using the modified Gleason grading $[13,15,17,18,28]$. Some found an improved concordance between biopsy and RP specimen Gleason score from $31.9 \%$ to $44.3 \%$ [17] and from $58 \%$ to $72 \%$ [15], while others found either a marginal improvement from $64.1 \%$ to $69.9 \%$ [18], no significant improvement [13] or agreement worsened from $67 \%$ to $58 \%$ [28]. In the study by Helpap et al. [15] Gleason score 7 increased from $36 \%$ to $83 \%$ in RP specimens and it is thus not surprising that the grade concordance was improved.

There are several problems with grade inflation. Comparisons with historical datasets will be difficult and this undermines the utility of previous research. More important, the prognostic impact of a certain grade changes, which has clinical implications. Theoretically, the outcome will improve in each grade category [9]. This causes considerable confusion among urologists when using grade data for treatment planning. By upgrading, a substantial number of tumours previously classified as low-risk are today considered intermediate risk. This has clinical consequences as risk category classification affects which type of therapy is offered. A follow-up study based on NPCR data showed that patients aged $<70$ years with low-risk tumours on AS had a risk of dying from prostate cancer of only $2.4 \%$ within 10 year after diagnosis [36]. It is likely that more patients would have been eligible for AS with earlier grading practices.

The true test of validity of the new Gleason system is its correlation with patient outcome. Because of too short a follow-up period, few studies have been done on the correlation between modified Gleason score and outcome. Billis et al. [14] claimed that the modified Gleason score was a better predictor of biochemical recurrence after RP than the conventional Gleason score. Yet, Kaplan-Meier curves of modified Gleason score 6 and 7 were almost identical and only the small group of Gleason score 8-10 had a worse outcome. In a small study by Uemura et al. [18] the modified Gleason score in needle biopsies was a stronger predictor of biochemical recurrence-free survival after RP than conventional Gleason score. However, in a large study Delahunt et al. [27] recently found that both scoring models predicted nadir PSA after radiotherapy, but conventional Gleason score outperformed modified Gleason score.

It is problematic that 6 years after the ISUP consensus meeting the upgrading trend still does not seem to be slowing down. It is a very important task for the pathology community to establish criteria for standardisation of detection thresholds for the identification of high-grade patterns. There was a recent attempt by the European Network of Uropathology, who distributed reference images for testing and calibration of 337 European pathologists [37]. More large-scale image libraries would help to establish a 'gold standard' for 
contemporary grading. Gleason grading is likely to remain a central instrument for guidance of the treatment decision and quality assurance of this measure is therefore of utmost clinical importance. Grading systems need to be as stable as possible to enable comparisons over time and to facilitate the interpretation of the prognostic impact of grade. This is best achieved by balancing the need of changes imposed by improved understanding of tumour biology against a conservative approach.

\section{Conflict of Interest}

None declared.

\section{References}

1 Albertsen PC, Hanley JA, Gleason DF, Barry MJ. Competing risk analysis of men aged 55 to 74 years at diagnosis managed conservatively for clinically localized prostate cancer. JAMA 1998; 280: 975-80

2 Delahunt B, Miller RJ, Srigley JR, Evans AJ, Samaratunga H. Gleason grading: past, present and future. Histopathology 2012; 60: 75-86

3 Gleason DF. Classification of prostatic carcinomas. Cancer Chemother Rep 1966; 50: 125-8

4 Pierorazio PM, Walsh PC, Partin AW, Epstein JI. Prognostic Gleason grade grouping: data based on the modified Gleason scoring system. BJU Int 2013; 111: 753-60

5 Epstein JI, Allsbrook WC Jr, Amin MB, Egevad L. The 2005 International Society of Urological Pathology (ISUP) consensus conference on Gleason grading of prostatic carcinoma. Am J Surg Pathol 2005; 29: 1228-42

6 Egevad L, Allsbrook WC Jr, Epstein JI. Current practice of Gleason grading among genitourinary pathologists. Hum Pathol 2005; 36: 5-9

7 Epstein JI. An update of the Gleason grading system. J Urol 2010; 183: 433-40

8 Brimo F, Montironi R, Egevad L et al. Contemporary grading for prostate cancer: implications for patient care. Eur Urol 2013; 63: 892-901

9 Albertsen PC, Hanley JA, Barrows GH et al. Prostate cancer and the Will Rogers phenomenon. J Natl Cancer Inst 2005; 97: 1248-53

10 Ghani KR, Grigor K, Tulloch DN, Bollina PR, McNeill SA. Trends in reporting Gleason score 1991 to 2001: changes in the pathologist's practice. Eur Urol 2005; 47: 196-201

11 Gilliland FD, Gleason DF, Hunt WC, Stone N, Harlan LC, Key CR. Trends in Gleason score for prostate cancer diagnosed between 1983 and 1993. J Urol 2001; 165: 846-50

12 Smith EB, Frierson HF Jr, Mills SE, Boyd JC, Theodorescu D. Gleason scores of prostate biopsy and radical prostatectomy specimens over the past 10 years: is there evidence for systematic upgrading? Cancer 2002; 94: 2282-7

13 Zareba P, Zhang J, Yilmaz A, Trpkov K. The impact of the 2005 International Society of Urological Pathology (ISUP) consensus on Gleason grading in contemporary practice. Histopathology 2009; 55: 384-91

14 Billis A, Guimaraes MS, Freitas LL, Meirelles L, Magna LA, Ferreira U. The impact of the 2005 international society of urological pathology consensus conference on standard Gleason grading of prostatic carcinoma in needle biopsies. J Urol 2008; 180: 548-53

15 Helpap B, Egevad L. The significance of modified Gleason grading of prostatic carcinoma in biopsy and radical prostatectomy specimens. Virchows Arch 2006; 449: 622-7

16 Kuroiwa K, Uchino H, Yokomizo A, Naito S. Impact of reporting rules of biopsy Gleason score for prostate cancer. J Clin Pathol 2009; 62: 260-3
17 Ozok HU, Sagnak L, Tuygun C et al. Will the modification of the Gleason grading system affect the urology practice? Int J Surg Pathol 2010; $18: 248-54$

18 Uemura H, Hoshino K, Sasaki T et al. Usefulness of the 2005 International Society of Urologic Pathology Gleason grading system in prostate biopsy and radical prostatectomy specimens. BJU Int 2009; 103: $1190-4$

19 Carter HB. Management of low (favourable)-risk prostate cancer. BJU Int 2011; 108: 1684-95

20 Dall'Era MA, Albertsen PC, Bangma C et al. Active surveillance for prostate cancer: a systematic review of the literature. Eur Urol 2012; 62: 976-83

21 Etzioni R, Penson DF, Legler JM et al. Overdiagnosis due to prostate-specific antigen screening: lessons from U.S. prostate cancer incidence trends. J Natl Cancer Inst 2002; 94: 981-90

22 Mohler J, Bahnson RR, Boston B et al. NCCN clinical practice guidelines in oncology: prostate cancer. J Natl Compr Canc Netw 2010; 8: 162-200

23 Tosoian JJ, Trock BJ, Landis $\mathbf{P}$ et al. Active surveillance program for prostate cancer: an update of the Johns Hopkins experience. J Clin Oncol 2011; 29: 2185-90

24 Albertsen PC, Hanley JA, Penson DF, Barrows G, Fine J. 13-year outcomes following treatment for clinically localized prostate cancer in a population based cohort. J Urol 2007; 177: 932-6

25 Albertsen PC, Hanley JA, Fine J. 20-year outcomes following conservative management of clinically localized prostate cancer. JAMA 2005; 293: 2095-101

26 Van Hemelrijck M, Wigertz A, Sandin F et al. Cohort Profile: the National Prostate Cancer Register of Sweden and Prostate Cancer data Base Sweden 2.0. Int J Epidemiol 2013; 42: 956-67

27 Delahunt B, Lamb DS, Srigley JR et al. Gleason scoring: a comparison of classical and modified (international society of urological pathology) criteria using nadir PSA as a clinical end point. Pathology 2010; 42: $339-43$

28 Morales C, Margel D, Yap S et al. Impact of the 2005 ISUP Gleason scoring system modification on our ability to predict extraprostatic extension. J Urol 2012; 187: e661

29 Carter HB, Partin AW, Walsh PC et al. Gleason score 6 adenocarcinoma: should it be labeled as cancer? J Clin Oncol 2012; 30: 4294-6

30 Jang TL, Han M, Roehl KA, Hawkins SA, Catalona WJ. More favorable tumor features and progression-free survival rates in a longitudinal prostate cancer screening study: PSA era and threshold-specific effects. Urology 2006; 67: 343-8

31 Egevad L, Engström K, Mattson S, Wester K, Busch C. Heterogeneity of DNA ploidy in prostate cancer. J Urol Pathol 1999; 10: 23-37

32 Iczkowski KA, Torkko KC, Kotnis GR et al. Digital quantification of five high-grade prostate cancer patterns, including the cribriform pattern, and their association with adverse outcome. Am J Clin Pathol 2011; 136: 98-107

33 Egevad L, Norlen BJ, Norberg M. The value of multiple core biopsies for predicting the Gleason score of prostate cancer. BJU Int 2001; 88: $716-21$

34 Inoue LY, Trock BJ, Partin AW, Carter HB, Etzioni R. Modeling grade progression in an active surveillance study. Stat Med 2014; 33: 930-9

35 Egevad L. Reproducibility of Gleason grading of prostate cancer can be improved by the use of reference images. Urology 2001; 57: 291-5

36 Stattin P, Holmberg E, Johansson JE, Holmberg L, Adolfsson J, Hugosson J. Outcomes in localized prostate cancer: National Prostate Cancer Register of Sweden follow-up study. J Natl Cancer Inst 2010; 102: 950-8

37 Egevad L, Ahmad AS, Algaba F et al. Standardization of Gleason grading among 337 European pathologists. Histopathology 2013; 62: $247-56$ 
Correspondence: Lars Egevad, Department of Oncology-Pathology, Karolinska Institutet, Radiumhemmet P1:02, Karolinska University Hospital, 17176 Stockholm, Sweden.

e-mail: lars.egevad@ki.se
Abbreviations: AS, active surveillance; ISUP, International Society of Urological Pathology; NPCR, National Prostate Cancer Register; OR, odds ratio; RP, radical prostatectomy; s-PSA, serum levels of PSA. 